DOI: 10.11606/issn.1676-6288.prolam.2016.119747

\title{
POLITICA EXTERIOR DE VENEZUELA PARA AMÉRICA LATINA DURANTE EL GOBIERNO DE HUGO CHÁVEZ ${ }^{1}$
}

\section{VENEZUELAN FOREIGN POLICY FOR LATIN AMERICAN DURING HUGO CHAVEZ GOVERNMENT}

\author{
Ana Sofia Garcia Salas ${ }^{2}$ \\ Universidade de São Paulo, São Paulo (SP), Brasil.
}

\begin{abstract}
Resumen: Estudiamos la política exterior de Venezuela para América Latina desde 1999 hasta 2013 con el objetivo de comprender los cambios introducidos por Hugo Chávez en las relaciones internacionales del país. En este trabajo se reconocen dos etapas del gobierno de Hugo Chávez: una de relativa continuidad de las políticas puntofijistas que transcurre entre 1999 y 2004, y una de absoluta novedad, que va desde 2004 hasta 2013, donde las alianzas políticas con países ideológicamente a fin, fue la principal estrategia de política exterior.
\end{abstract}

Palabras Claves: Venezuela; ALBA; PETROAMERICA.

\begin{abstract}
We study the foreign policy of Venezuela for Latin America from 1999 to 2013 with the aim of understanding the international relations changes, introduced by Hugo Chavez, in the country. In this paper, are recognized two stages of Hugo Chavez: the first one, relative continuity of puntofijista policies that elapses between 1999 and 2004, and the second one an absolute novelty, which runs from 2004-2013, where political alliances with countries ideologically related it was the main foreign policy strategy.
\end{abstract}

Key Words: Venezuela; ALBA; PETROAMERICA.

1 El trabajo presenta los resultados principales de la tesis de maestría titulada Política externa de Venezuela para América Latina durante el gobierno de Hugo Chávez, defendida en el Programa de pós-graduacao em Integracao da América Latina de la Universidad de Sao Paulo en junio de 2016, bajo la orientación del Profesor Doctor Renato Seixas.

2 Mestra por el Programa de pós-graduacao em Integracao da América Latina de la Universidad de Sao Paulo (PROLAM/USP). E-mail:<anasofiagarciasalas@gmail.com>. Recibido en: 01.03.2016; aceptado en: 14. 07. 2016. 


\section{INTRODUCCIÓN}

Un aspecto que diferencia a Venezuela del resto de los países productores de hidrocarburos de la región, es el carácter medular que representa la renta del petróleo en su economía.

Por su pasado como colonia, Venezuela heredó la tradición española que el Estado es dueño del suelo y del subsuelo del territorio nacional. En un primer momento, cuando empezó la prospección de la venta del petróleo a escala internacional, los gobiernos venezolanos negociaban una mayor participación en las ganancias de la renta petrolera.

Posteriormente en 1976, cuando se nacionalizó la industria, el Estado venezolano adquirió un carácter de Estado petrolero. Pasando a administrar la industria y continuando con la repartición de la renta, lo que permitió la existencia de un capitalismo de Estado.

Esta realidad macro económica y la naturaleza del negocio petrolero, hicieron con que los diferentes gobiernos desenvolvieran estrategias para vender el producto en el mercado internacional.

Las estrategias a las que hacemos mención, como es evidente, siempre fueron vulnerables a las coyunturas internacionales, a los acontecimientos nacionales y hasta a las personalidades de los mandatarios.

Mismo así es reconocido que durante la vigencia del Pacto de Punto Fijo ${ }^{3}$ se mantuvieron algunos lineamientos base. (ROMERO, 2002), identifica al menos tres hilos conductores del sistema democrático pactado en su aspecto internacional: el nacionalismo antiimperialista, el americanismo y la democratización internacional. Este último figura con especial atención y concibe la democracia representativa como el sistema político insustituible en la comunidad de naciones, sobre todo en el territorio latinoamericano (ROMERO, 2002, p.176).

Entonces, del estudio de la historia política del país queda evidente que desde la época de Bolívar Venezuela reivindica su identidad latinoamericanista y manifiesta su interés en cooperar para la resolución de problemas comunes. Con la llegada de los regímenes democráticos encontramos una excepción de esta realidad, la Doctrina Betancourt ${ }^{4}$. Es por eso que podemos afirmar que desde el mandato de Raúl Leoni (1964-1969) la cultura política del país le otorga un trato especial a la integración con los demás países de la región.

3 El Pacto de Punto Fijo, fue un pacto entre los partidos políticos venezolano de respeto al orden democrático, es decir, los actores políticos se comprometieron a no intentar llegar al poder por la vía de la ruptura del orden democrático. Juan Carlos Rey Martínez lo denominó de Sistema Populista de Conciliación de Elites (REY 1972; REY 1989; REY 1991, apud ROMERO, 2015).

4 Iniciativa central de la política exterior venezolana entre 1958-1964, la cual predicaba la ruptura de relaciones diplomáticas con regímenes de facto que hubiesen derrocado gobiernos constitucionales, procurando desalentar proyectos golpistas mediante la amenaza del aislamiento internacional, tanto en el país, como en el resto de América Latina. Dado sus efectos aislacionistas y su carácter parcialmente intervencionista, su aplicación quedo sin efecto al poco tiempo. El mismo Rómulo Betancourt (ex presidente creador de la doctrina) reconoce que "Venezuela se quedó virtualmente sola en el continente ante una marea de golpes de Estado contra gobiernos constitucionales" (BETANCOURT, 1968, p. 70). 
Ahora bien, con el agotamiento del sistema político de la democracia pactada y consecuentemente, con la llegada de Hugo Chávez Frías a la presidencia República, se abre un nuevo ciclo en la historia política venezolana.

No obstante, durante los primeros años del mandato chavista se observa una relativa continuidad de las políticas puntofijistas. La ruptura de esa fase la podemos ubicar en el año 2004. Cuando realmente mudan los paradigmas que regían la conducta del país en el escenario internacional.

La política exterior de Venezuela durante el mandato de Hugo Chávez (1999-2013), especialmente la estrategia integracionista con los demás países de América Latina constituye el objetivo principal de esta investigación, el cual estudiaremos a través de los documentos oficiales donde reposa la estrategia internacional de convertir a Venezuela en el país líder de la integración latinoamericana.

Partimos de la hipótesis que la estrategia chavista en materia internacional es un reflejo de su sistema de desarrollo doméstico. Consideramos también que el hecho que sea una concepción con un ideal político determinado, genera dudas sobre su sustentabilidad. Sobre todo, porque deja poco espacio para la articulación con otros actores internacionales con una concepción de mundo diferente.

En las páginas siguientes intentaremos verificar ¿Cuáles fueron los cambios introducidos por Hugo Chávez en las relaciones internacionales de Venezuela? y ¿Qué papel juega el petróleo en la estrategia integracionista propuesta por el gobierno venezolano?

\section{DE PUNTO FIJO AL CHAVISMO (1958-1999)}

Antes de entrar al estudio de la política exterior chavista, es necesario conocer los antecedentes que formaron la identidad de Venezuela como actor internacional. Consideramos importante hacer algunas precisiones en virtud de la reiterada afirmación del líder del proyecto socialista de romper con los parámetros en materia internacional constituidos por los gobiernos puntofijistas.

\subsection{ASPECTOS HISTÓRICOS RELEVANTES}

Del análisis histórico sobre la política externa de Venezuela, se verifica que el país a inicios del siglo XX, a pesar de haberse constituido uno de los centros irradiadores del proceso de independencia latinoamericano, tuvo una diplomacia influenciada por su pasado colonial. A principios de los años 1900 no había constituido aún, su Estado nación. Con una populación analfabeta y desigualmente distribuida, trabajaba en una precaria economía primario exportadora, en cuanto el caudillismo y las luchas internas marcaban la vida de la elite agraria y militar (VIZENTINI, 2001, p. 56). 
En el estudio percibimos que en ese período el país no tenía vinculaciones internacionales claramente definidas. Sin embargo, es en la dictadura de Juan Vicente Gómez (1908-1935) que se ubica el inicio de la prospección petrolera venezolana ligada al capital internacional. En ese entonces se comienza a construir la infraestructura viaria y con esto el Estado fue adoptando una administración cada vez centralizada.

Tras la muerte de Juan Vicente Gómez (1935) el país vive un corto período democrático donde se consiguieron algunos avances importantes en materia de legislación petrolera. A través de los tributos, el Estado consiguió una retribución mayor (y más justa) de las riquezas generadas por la venta del recurso natural. En este particular resaltan el gobierno de Isaías Medina Angarita; con la reforma petrolera de 1943 y el cortísimo mandato de Rómulo Gallegos con el fisty-fisty. No obstante, con la llegada en 1948 de la dictadura militar de Marcos Pérez Jiménez hubo un retroceso respecto a la justa distribución de las ganancias petroleras (TORO, 1992, pp. 01-05).

En nuestra opinión, el retroceso de los avances nacionalistas en materia petrolera, mostró al país como un lugar donde las inversiones extranjeras podían estar seguras. El reflejo de esto lo encontramos en el escenario económico; dos años después de la llegada de Pérez Jiménez, para los años 50, Venezuela había alcanzado el puesto de primer país productor y segundo exportador mundial de petróleo, lo que llevó a la estabilidad en las relaciones entre los distintos sectores de la elite socio-económica; dejando así, completamente de lado, la economía de agricultura tropical que compartía con los demás países del Caribe.

En 1958 el régimen militar de Pérez Jiménez fue derrocado y, se comienza a estructurar el paradigma que dominó el sistema político venezolano durante la década de los 60, El Pacto de Punto Fijo. Juan Carlos Rey Martínez lo denominó de Sistema Populista de Conciliación de Elites (REY 1972; REY 1989; REY 1991, apud ROMERO, 2015). Según este autor, el sistema político venezolano, en ese momento, estaba fundamentado en la presencia de dos partidos democráticos mayoritarios, Acción Democrática AD (socialdemócrata) y COPEI (socialcristiano), un fuerte respaldo económico por parte de un Estado petrolero, una economía rentista, la neutralidad de las Fuerzas Armadas, un reconocimiento internacional a la democracia representativa y un apoyo mayoritario social, tanto del sector empresarial como de las clases medias y los sectores obreros y campesinos.

Este enfoque no obtuvo el apoyo unánime de todos los sectores políticos y académicos venezolanos. Desde la izquierda, hubo quienes alertaron que el modelo venezolano no era más que otro ejemplo latinoamericano del reformismo y que en verdad lo que se quería construir era un modelo que ocultara la dependencia del país del capitalismo internacional, especialmente de Estados Unidos. Desde la óptica de algunos sectores conservadores, se estimaba que Venezuela no cumplía con las condiciones mínimas para desarrollar el modelo democrático y que por lo tanto se requería de un gobierno autoritario para lograr el desarrollo. Finalmente, estaban aquellos seguidores del pensamiento liberal quienes propugnaban un régimen de libre empresa para Venezuela y la reducción del peso del populismo y del Estado (ROMERO, 2011; ROMERO, 2015).

Lo cierto es que bajo el paradigma del Pacto de Punto Fijo, transcurrieron los primeros cuarenta años democráticos de la República 1958-1998. 
Durante este tiempo el marco legal institucional fue la constitución de 1961, la cual establecía en su preámbulo el conjunto de principios generales que regirían la conducta del país en materia internacional. Juan Carlos Rey Martínez hace una síntesis sobre su percepción sobre la actuación de Venezuela en el escenario internacional y resalta principios como el carácter pacífico del país, la búsqueda de la integración económica, el deber de cooperar internacionalmente, la promoción de la democracia y otros conceptos emanados de la Carta de las Naciones Unidas; la discrecionalidad del presidente de la República en el ámbito de la política exterior; el carácter petrolero de la nación, pero en función de promotor de una economía diversificada, y el desarrollo de varias identidades para una política exterior considerada a su vez como: andina, caribeña, hemisférica, tercermundista y amazónica (REY, 1983, apud ROMERO, 2002, p. 43).

\subsection{VENEZUELA COMO ACTOR INTERNACIONAL}

Venezuela es un país democrático, que ha mostrado, tanto en su Constitución como en la práctica, su vocación para implantar y defender la democracia representativa dentro y fuera de su territorio (GUERÓN, 1989, p. 351). Contar con un sistema democrático sólido llegó a ser un pre requisito para la instalación de relaciones diplomáticas con Venezuela. La defensa de la democracia representativa sólo dejo de ser la protometa de la política exterior del país, una vez que su consolidación ya no representaba un reto, era una realidad.

Un país que se identifica con las mayorías del Tercer Mundo por compartir rasgos socioeconómicos del subdesarrollo pero que, como productor de petróleo pertenece a una minoría privilegiada en ingresos e influencias (GUERÓN, 1989, p. 351). Desde los años 30, los gobiernos venezolanos han tenido que lidiar con esta dualidad. Ese escenario fue propicio para que los gabinetes ejecutivos formularan y ejecutaran exitosamente políticas de tilde nacionalista, con el objetivo de aumentar la apropiación de los recursos provenientes de la venta del petróleo venezolano.

Es también, un país americano que comparte la herencia de América Latina; constitucionalmente comprometido a favorecer la integración latinoamericana pero que, además, pertenece a la zona andina, al Caribe y a la sub región amazónica.

De estas características consideradas como permanentes se desprenden los grandes temas y orientaciones del país en el ámbito internacional.

Mismo así reconocemos que estas múltiples identidades y/o características, desde 1958, circunstancialmente, han logrado un mayor grado de conexión con la cultura política, las agendas de política exterior y las actuaciones internacionales del país (GUERÓN, 1989, p. 351).

Así pues, la búsqueda de la estabilidad democrática que privilegio la identidad de Venezuela como país democrático y occidental, constituyó, durante los gobiernos de los presidentes Rómulo Betancourt (1959-1954), Raúl Leoni (1964-1969) y el comienzo del primer gobierno de Rafael Caldera (1969-1974) el hilo conductor de la política exterior venezolana. 
En una especie de ciclo de ampliación y diversificación de vínculos entre el juego doméstico y el mundial, se destaca la identidad venezolana como país en desarrollo y alcanza su punto máximo durante la primera Presidencia de Carlos Andrés Pérez (1974-1979) cuando la promoción de un nuevo orden económico internacional expresaba, en términos muy ambiciosos y de enormes expectativas, las aspiraciones tercermundistas de lograr la inserción favorable de Venezuela en la economía mundial, utilizando el petróleo como palanca.

Los cambios en el escenario mundial, regional y doméstico hicieron con que los gobiernos de Luis Herrera Campins (1979-1984) y Jaime Lusinchi (1984-1989) redujeran significativamente la agenda internacional venezolana como consecuencia de la presión del endeudamiento externo, los conflictos centroamericanos y las crisis regionales como la Guerra de las Malvinas (1982) y la intervención Norteamericana a Granada. Disminuyendo la intensidad de la presencia internacional y enfocando su acción principalmente hacia la subregión del Caribe. Las dos características destacadas en este momento fueron: la de país americano y deudor.

Durante los años noventa, los autores reconocen una pérdida de referencias claras para la política exterior, y con ello, el final de un ciclo marcado por un modelo político económico populista-estatista, y por un ambiente internacional relativamente estable y de baja complejidad. Con la acelerada expansión del capitalismo, la caída del muro del Berlín, la revolución tecnológica y las políticas económicas de liberación de mercados; las relaciones entre los actores políticos, sociales y económicos mudaron, a nivel mundial, regional y local.

Así durante los mandatos de Carlos Andrés Pérez y Rafael Caldera 1989-1993; 19941998, respectivamente, se cierra el ciclo de la política exterior de la democracia pactada en el país. Cediendo pasó en la vida política venezolana a un militar que prometía romper con la forma y los métodos empleados por los gobiernos anteriores, los puntofijistas.

Entonces, del estudio pudimos constatar que durante los primeros 40 años democráticos de la República existió una gestión internacional con un conjunto de fines, intereses, objetivos, acciones y reglas del juego permanentes de carácter internacional. Evidentemente, los cambios coyunturales que tuvieron lugar dentro del sistema internacional y nacional; marcados por la creciente complejidad experimentada por las organizaciones del sistema político internacional y la constante influencia ejercida por la personalidad e intereses de los decisores y encargados de la política exterior, le imprimieron a cada mandato su lógica, incoherencias y discontinuidades.

Como mencionamos en la parte introductoria, nos apegamos a la concepción de María Teresa Romero, cuando reconoce al menos tres hilos conductores del sistema democrático pactado en su aspecto internacional: el nacionalismo antiimperialista, el americanismo y la democratización internacional (ROMERO, 2002, p. 174).

Reconocemos que el nacionalismo antiimperialista está estrechamente relacionado, con la concepción económica que apela por aquellas políticas de intervencionismo estatal o de economía dirigida y planificada por el Estado en los procesos de producción y distribución de la riqueza nacional.

La idea de americanista desde nuestro entender tiene dos frentes: Cuando se previa una amenaza extra continental, se tendía a enfatizar en la propuesta interamericana de defensa 
continental; cuando, sin embargo, se percibía la amenaza de intervención por parte de la propia potencia del norte, se hacía hincapié en el entendimiento e integración latinoamericana.

En el proyecto internacional puntofijista, figura con especial atención la idea de democratización internacional que concibe a la democracia representativa como el sistema político insustituible en la comunidad de naciones sobre todo en territorio latinoamericano (ROMERO, 2002, p. 176).

Además agregamos que, Luego de la Doctrina Betancourt y el Pentágono Petrolero 5 , no se verifica en la política exterior de Venezuela otra agenda de política exterior contentiva de un plan de Estado nacional (no de gobierno) con objetivos concretos que alcanzar a mediano o largo plazo.

También observamos que la participación de la sociedad civil y otros agentes políticos (partidos, empresa privada, entre otros), en la formulación de estás agendas internacionales, si en algún momento tuvieron espacio, el mismo se fue extinguiendo con la sobredimensión que ha ido adquiriendo el Estado venezolano.

Por último se hace necesario recalcar que el elemento común de los gobiernos que han pasado por la casa presidencial venezolana, es la utilización del petróleo como herramienta de inserción del país en la economía internacional.

La razón de esto la encontramos en el hecho que, como país exportador de petróleo, Venezuela depende de este recurso en lo económico, y en lo político. Como bien lo explica Eva Josko de Guerón, los ingresos petroleros financian tanto los proyectos de desarrollo económico como los programas distributivos del Estado. El lucro proveniente de dicho ingreso único, ha sido un factor de fundamental importancia en la estabilización del sistema político, pues -continua explicando la autora- ha permitido el reparto de beneficios sin graves conflictos en torno a la distribución (GUERÓN, 1989, p. 354).

\section{LA POLÍTICA EXTERIOR DE HUGO CHÁVEZ}

Entonces, con la llegada de Hugo Chávez a la presidencia de Venezuela el 02 febrero de 1999, se comienza a cerrar el ciclo de relativa continuidad de políticas imperantes los durante los 40 años de democracia pactada (1958-1999). El mismo día de su juramentación, el nuevo presidente manifestó su voluntad de impulsar las "[...] transformaciones democráticas

5 Juan Pablo Pérez Alfonso, quien fue Ministro de Minas del gobierno de Rómulo Betancourt formuló una agenda política internacional cuyo fin era el aumento de la presencia del Estado en el negocio petrolero, para esto se definió un plan conocido como el Pentágono Petrolero el cual postulaba cinco acciones que implementar: 1) lograr la participación razonable y con superioridad jerárquica del Estado venezolano en la industria petrolera; 2) la ejecución de la política de no más concesiones; 3 ) la "siembra del germen" de la futura industria petrolera estatal a través de la Corporación Venezolana del Petróleo (CVP); 4) el aumento del control del Estado sobre las actividades de la industria, fiscalizando precios y volúmenes de producción, a través de la Comisión Coordinadora de la Conservación de los Hidrocarburos (CCCCH); y 5) La creación de la Organización de los Países Exportadores de Petróleo (OPEP). 
necesarias para que la República nueva tenga una Carta Magna adecuada a los nuevos tiempos." (CHÁVEZ, 1999).

De acuerdo con Carias (2000), las transformaciones a las que se refería Hugo Chávez respondían a la ideologización de un mundo socialista, y retaban la idea de mundo liberal que caracterizó la década de los noventa.

Así pues, el proyecto bolivariano procurando romper con la pasividad histórica de la República venezolana frente a la subordinación de intereses geopolíticos del imperialismo norteamericano, se planteó como objetivo, construir una nueva geopolítica internacional a través de la creación de múltiples polos de poder, conformando bloques regionales articulados en función de servir de contra peso a la influencia que los Estados Unidos de Norte América ejerce sobre nuestro continente y el resto del mundo. Defendiendo que los principios de amistad, solidaridad y cooperación entre las naciones, deben imperar por ante los intereses económicos en las relaciones internacionales (CHÁVEZ, 2001, pp. 07-09).

Esa estrategia de política exterior estaba compuesta de varias fases: la primera que va entre el año 1999 y 2010, denominada en el Plan de Desarrollo Económico de la Nación la época de plata; sus formuladores consideraron este período como una etapa de transición hacia la época de oro que transcurría entre los años 2011 y 2020, en la cual acontecería la realización de la Revolución Bolivariana (CHÁVEZ, 2001, p. 09).

La formulación de una agenda de gobierno cuya ejecución está prevista por un período de 20 años, resultó un hecho sin precedentes en la historia política del país, y como es lógico, despertó la atención de diversos sectores de la sociedad venezolana; académicos, medios de comunicación, gremios profesionales, partidos políticos tradicionales y empresarios privados. Sin embargo estos primeros años transitorios del Gobierno de Hugo Chávez no representaron una ruptura abrupta respecto a la actuación internacional que el país mantuvo durante la vigencia del Pacto de Punto Fijo.

No ocurre lo mismo respecto a la formulación de agendas internacionales y los principios constitucionales a los que éstas responden. A diferencia de la constitución de 1961; que priorizaba sustentar el orden democrático como único e irrenunciable medio de asegurar los derechos y la dignidad de los ciudadanos. La Constitución de 1999 pretende refundar la República para establecer una sociedad democrática, participativa y protagónica, multiétnica y pluricultural (ROMERO; ROMERO; CARDOZO, 2003, p. 166).

A diferencia de los gobiernos puntofijistas que tenían como meta principal (cada uno con sus matices) la defensa a la democracia representativa, el proyecto de la revolución socialista concibe la democracia como participativa y protagónica. Este cambio de concepción sobre el orden democrático, representa la ruptura de uno de los principales lineamientos que guiaron la política exterior del país desde la llegada de los regímenes electos a través del voto popular. Este hecho, como lo coloca (CARÍAS, 2000, p. 3), responde a la ideologización de un mundo socialista.

Dentro de esa lógica, la primera agenda internacional del gobierno socialista, hacia la Revolución Bolivariana, establece como principal objetivo fortalecer la soberanía nacional e 
impulsar la multipolaridad de la sociedad internacional con el fin de equilibrar las relaciones económicas entre los diversos países del mundo (PND, 2001, p. 155).

Para esto, como se evidencia del documento oficial objeto de estudio, el gobierno considera necesario, fortalecer el posicionamiento de Venezuela en la economía internacional, mediante la consolidación y diversificación de las relaciones diplomáticas, visando promover la integración regional en diversas áreas, inclusive la militar (PND, 2001, p. 155-152).

Esa agenda formulada dos años después de la llegada del partido chavista al poder, deja claras las ambiciones internacionales que se planteaba la revolución bolivariana. No obstante como mencionamos, la misma representa una primera fase de reconocimiento que antecedió a la construcción del mundo multipolar, una etapa más arriesga y radical.

En el año 2004 ubicamos esa etapa, en la que el país efectivamente se alejó prácticas utilizadas por los gobiernos puntofijistas, que por medio de sus agendas de política exterior, procuraban la inserción de Venezuela en el sistema económico internacional como proveedor seguro de energía de los países desarrollados.

Sin embargo esta fecha mucho se adelanta al período establecido en el Plan Económico y Social de la Nación 2001-2007, en el cual la etapa de construcción del Socialismo del Siglo XXI estaba prevista para el año 2010.

Asociamos la acelerada construcción del proyecto socialista, con el de golpe de Estado que sufrió el gobierno en el año 2003 por parte de los sectores más conservadores de la vida política del país, y tras el triunfo del referéndum revocatorio del mandato presidencial en el 2004.

Consideramos que estos acontecimientos en el ámbito doméstico, hicieron con que el presidente venezolano reflexionara sobre sus estrategias políticas en general y priorizara la consolidación del proyecto bolivariano.

Como consecuencia, el 12 y 13 de noviembre del 2004 fue celebrado en la ciudad de Caracas, el Taller de Alto Nivel Estratégico donde asistieron los gobernadores (as), alcaldes (as), diputados (as), dirigentes del partido político Movimiento Quinta República (MVR), el alto mando militar, dirigentes sociales, ministros (as) y el vicepresidente.

En el transcurso de estos dos días, Hugo Chávez junto con los otros líderes del proyecto socialista bolivariano buscaron " [...] elaborar un instrumento general acerca de lo que llamo la nueva etapa a la que hemos entrado [...]" (CHAVEZ, 2004, p. 12), haciendo referencia al proyecto socialista, que aún estaba en sus primeros pasos.

Pero estos nuevos lineamientos estaban lejos de ser una improvisación por causa de los acontecimientos domésticos. Tal como comenta el máximo líder de la revolución socialista, los nuevos paradigmas de la política venezolana son resultado de la evolución del plan de acción formulado para conseguir el derrocamiento del gobierno de Carlos Andrés Pérez el 04 de febrero de 1992. 
[...] en 1994, pocos meses después de haber salido de prisión, nosotros teníamos un mapa estratégico. Estoy seguro que aquí hay algunos que lo recuerdan, y pudieran hasta explicarlo. Yo de tanto repetirlo y explicarlo por el país, en tantas reuniones que hicimos me lo sé de memoria. Ese mapa fue el producto de una serie de trabajos y reuniones donde estaban el hoy ministro Giordani [...], el ex ministro Héctor Navarro, y un grupo de militares y civiles... Con ese mapa navegamos en el 94, en el 95 y en el 96. En el 96 empezamos hacerle adecuaciones... Entonces... en el 97 se abrió la ventana electoral de 1998 y decidimos irnos por ahí. Alguna gente de nuestras filas se fue sino molesta, desencantada; esa gente que siempre cree en la lucha armada pues. (CHAVEZ, 2004, p. 12).

El instrumento general con el que "navegaría" la Revolución Bolivariana en su nueva etapa, quedó conocido como el Nuevo Mapa Estratégico y fue explicado en dos sesiones por el mismo Hugo Chávez. Su intervención durante el Taller de Alto Nivel Estratégico, fue sistematizada y organizada por Harnecker (2004) quien, en la versión impresa, suprimió repeticiones y datos de menor interés.

Este excelente trabajo, hace las veces de documento en el que reposa la exposición sobre la nueva etapa en la que estaría entrando el sistema político, económico y social venezolano.

Por esta razón ése trabajo junto con el Plan Nacional de Desarrollo 2001-2007 (PND) son los documentos de estudio donde buscamos la respuesta sobre cuáles son los cambios introducidos por Hugo Chávez en las relaciones internacionales de Venezuela.

\subsection{HACIA LA REVOLUCIÓN BOLIVARIANA}

Entonces, al comprar los principios, procedimientos y temas de la política exterior de la constitución de 1961 con los de la constitución de 1999 se evidencia que los dos textos constitucionales le otorgan un nivel de importancia estratégica a las relaciones internacionales.

Además, en materia de democracia y derechos humanos; relaciones económicas internacionales y seguridad, el texto de 1999 revela mayor elaboración y detalle en el tratamiento de lo internacional. No obstante, en el nuevo texto constitucional, se identifica la presencia de contradicciones en su elaboración orgánica, y especialmente de cambios en sus principios generales (ROMERO; ROMERO; CARDOZO, 2003, p. 166) (negrillas nuestras).

Si bien es cierto que, los primeros años de gestión de Hugo Chávez no representaron una ruptura abrupta de las agendas de política exterior de los gobiernos puntofijistas, no es menos cierto, que con la entrada en vigencia de la constitución de 1999, se pone en manifiesto el abandono del lineamiento principal de la política exterior del Estado venezolano durante la etapa de democracia pactada (1958-1998): la promoción y defensa de la democracia representativa, sobre todo, en el territorio latinoamericano y caribeño.

Ahora, de acuerdo con el preámbulo de la Constitución de 1999, el fin supremo del Estado es "[...] refundar la República para establecer una sociedad democrática, participativa y protagónica, multiétnica y pluricultural [...]”' (VENEZUELA, Constitución, 2000). 
En la nueva constitución no se menciona ni en el preámbulo ni en el apartado de las Relaciones Internacionales, que la promoción y defensa de la democracia representativa constituyan un lineamiento o fin de la política exterior, lo cual era contemplado en la constitución de 1961, en los siguientes términos: "[...] sustentar el orden democrático como único e irrenunciable medio de asegurar los derechos y la dignidad de los ciudadanos, y favorecer pacíficamente su extensión a todos los pueblos de la tierra"(VENEZUELA, Constitución, 1961).

La omisión de este principio tradicional, puede explicarse debido a la idea de democracia que guía la Constitución vigente (la participativa y protagónica); así, como por una concepción de mundo idealista de izquierda, que desafía la ideología liberal.

\subsection{PLAN NACIONAL DE DESARROLLO 2001-2007}

En la presentación del "primer plan de la nueva era constitucional bolivariana", se advierte que, aún cuando la norma constitucional limita la propuesta del Plan a una formulación de un período de seis años, la visión de desarrollo ahí establecida, contempla un mayor plazo. Este desacato constitucional, se justifica en el hecho de que la "[...] revolución venezolana se esté llevando a cabo en forma pacífica y democrática... y, que es por esta razón que tiene... un carácter y ritmo que se son propios [...]" (CHÁVEZ, 2001, p. 08).

Contrario al discurso que se sostenía en la Agenda Bolivariana de 2006, donde el ex presidente opinaba que el modelo de desarrollo copeyano venía acompañado de un ideal " [...] fetichista de libre mercado, libertad individualista y competencia, tras el cual se esconde la pretensión de recuperar y consolidar 'por los siglos de los siglos' la hegemonía de un modelo de acumulación [...]" (CHÁVEZ, 1996, p. 19). El sistema económico de desarrollo planteado en el PND está vinculado "[...] con un sistema productivo diversificado, competitivo, abierto hacia los mercados internacionales, basado en la iniciativa privada y con presencia del Estado en industrias estratégicas, pero con la apertura a la inversión privada [...]." (PND, 2001, p. 13).

Pero más allá de esa, y otras contradicciones, el Plan como un todo, en líneas generales, plantea la construcción de un nuevo modelo para Venezuela, el cual, según entendemos, servirá de base para la construcción del Socialismo del Siglo XXI.

Algo que queda evidente del documento en estudio es que si bien se reivindican valores nacionales y tercermundistas, así como las causas sociales de las clases menos favorecidas, en el mismo no existe posicionamiento respecto a una ideología política partidaria. Aún, cuando se hace mención a la segunda fase o época de oro de la revolución bolivariana, se omite la palabra socialismo y se utiliza el término de consolidación o realización de la revolución bolivariana.

Entonces, para hacer operativo este Plan, se establecieron cinco equilibrios cuya práctica se fundamenta en la participación protagónica de todo el pueblo venezolano sin discriminación alguna (PND, 2001, p. 15). Estos cinco equilibrios son: equilibrio económico, equilibrio social, equilibrio político, equilibrio territorial, equilibrio internacional. 
En lo que atinge al foco de estudio de esta investigación el equilibrio internacional, se establece que el objetivo principal de la política internacional es fortalecer la soberanía nacional y promover un mundo multipolar (PND, 2001, p. 156). Esta estrategia de pluralización multipolar prevé una serie de acciones a ser ejercidas en el ámbito internacional ${ }^{6}$.

En este período la identidad venezolana como país americano, en desarrollo y tercermundista presenta fuerte conexión con la agenda internacional objeto de estudio.

Consideramos que, se mantiene americanista por la vocación integracionista venezolana que data de los tiempos de Bolívar, sobre todo en la región de América Latina y el Caribe y, con la reafirmación de las relaciones internacionales con el socio económico por excelencia del país, Estados Unidos.

En relación a los bloques de integración se continúan las negociaciones que comenzó Rafael Caldera para el ingreso del país al MERCOSUR, y se propone la asociación de este bloque regional con la CAN, supeditando las negociaciones del ALCA a la previa conclusión de los acuerdos latinoamericanos (PND, 2001, p. 156).

La identidad como país en desarrollo la identificamos por procurar la inserción de Venezuela en la economía internacional capitalista a través de la exportación de meterías primas. Y la de tercermundista, por reivindicar el fortalecimiento de la cooperación Sur-Sur y la ampliación de las relaciones con otras regiones y países del mundo (PND, 2001, p. 156).

Otro aspecto que resalta y que se desarrolla de manera esquemática en el segundo documento que estudiaremos a continuación: el Proyecto Nacional Simón Bolívar- Primer Plan Socialista 2007-2013 (PPS) es: otorgarle carácter político a la integración para impulsar un nuevo modelo integracionista (PND, 2001, p. 156).

\subsection{EL NUEVO MAPA ESTRATÉGICO (2004-2006)}

Después de acontecimientos domésticos; como el golpe de Estado de 2002, accionado por los sectores más conservadores de la vida política del país y la ratificación en su cargo con el $59 \%$ del electorado a favor, Hugo Chávez con un demostrado apoyo popular empezó la acelerada construcción del Socialismo del Siglo XXI.

6 Intensificar el apoyo al proceso de pacificación regional; Promover la Integración Latinoamericana y caribeña, a través de la integración política como opción estratégica, impulsando un nuevo modelo de integración económica en América Latina y el Caribe; Consolidar y diversificar las relaciones internacionales por medio de la reafirmación las relaciones con los países vecinos y los socios económicos de Venezuela, el fortalecimiento de la cooperación SurSur y la ampliación de las relaciones con otras regiones y países del mundo; Fortalecer el posicionamiento de Venezuela en la economía internacional, afianzando la vigencia y la proyección de la OPEP a los fines de acelerar la internacionalización de la economía de Venezuela, procurando incrementar asociaciones estratégicas y promover un nuevo régimen de seguridad nacional hemisférico a través de la implementación de un nuevo modelo de la Fuerza Armada Nacional a los fines de fortalecer la defensa regional (PND, 2001, p. 156). 
Así entonces, convocó a los otros líderes de la revolución a un Taller de Alto Nivel Estratégico que procuraba, en palabras del fallecido ex presidente, enfocar el nuevo momento en el que se estaba "montado" la revolución bolivariana.

En ese evento fueron impartidas directrices que romperían definitivamente con la tradición política venezolana.

El nuevo mapa estratégico con el que "navegaría" la revolución bolivariana en su nueva etapa, fue explicado en dos sesiones por el "líder supremo" del proyecto socialista.

La intervención de Hugo Chávez en este evento "divisor de aguas", representa el testimonio de una propuesta aún no meramente radical de sus ideas, pero que constituye abono fértil para la maduración de reflexiones que en el presente siguen influenciando la tolda del gobierno chavista, aún después de su muerte.

El trabajo de Harnecker (2004), siguiendo el mismo orden esquemático propuesto por el fallecido ex Presidente, se divide en siete secciones ${ }^{7}$. Del texto se evidencia que el establecimientos de los grandes objetivos (sección medular del taller) fue la que más tiempo demando. De los 10 objetivos $^{8}$ ahí planteados, el último, seguir impulsando el nuevo sistema multipolar e internacional, es el que atinge nuestro foco de estudio. No obstante el mismo no fue desarrollado con detalle en el acto en cuestión.

Mismo así, nuestra intensión es resaltar que en ese acto se sientan las bases del nuevo modelo político, económico y social que comenzó a ser implementado en el país.

Por último no podemos dejar de mencionar que en ningún momento, los demás actores de interés como lo es: la sociedad civil organizada, la empresa privada, los partidos políticos de oposición, fueron invitados al acto en comento.

Por el contrario, la "nueva fase" de la revolución bolivariana no responde cabalmente a los principios de elaboración de políticas públicas. Es un plan concebido en la cabeza de pocas personas, entre ellas otros militares que acompañaron al ex presidente en el golpe de Estado. Es por esta razón que no existe una agenda como tal, si no lo que sirve de referencia es el trabajo de Harnecker (2004).

7. Un esbozo de la situación actual; 2. Enfoque del Nuevo Mapa Estratégico 3. La definición del Nuevo Mapa Estratégico; 4. Grandes Objetivos; 5. Elaboración de un plan preliminar de acción; 6. Elaboración de un cronograma de acción estratégica y 7. La designación del Entre coordinador del control y el monitoreo de los planes de acción.

8. Avanzar en la configuración de una nueva estructura social; 2. Articular y optimizar la nueva estrategia comunicacional; 3. Avanzar aceleradamente en la construcción del nuevo modelo democrático; 4. Acelerar la creación de la nueva institucionalidad del Estado; 5. Nueva estrategia integral contra la corrupción; 6. Desarrollar la nueva estrategia electoral; 7. Acelerar la construcción del nuevo modelo productivo rumbo a la creación del nuevo sistema económico; 8.Seguir instalando la nueva estructura territorial; 9.Profundizar y acelerar la configuración de una nueva estructura miliar nacional y 10 . Seguir impulsando el nuevo sistema multipolar e internacional. 


\section{VENEZUELA Y LA INTEGRACIÓN REGIONAL}

La estrategia para la creación de un sistema internacional multipolar se encuentra establecida en el Proyecto Nacional Simón Bolívar- Primer Plan Socialista de la Nación PPS (2007-2013).

Es en ése documento oficial, donde reposa la estrategia internacional de convertir a Venezuela en una potencia energética mundial a través de un bloque regional de poder que represente un quiebre para la hegemonía norteamericana, promoviendo así un orden mundial multipolar (PPS, 2007, p. 38-43).

La materialización de ésa estrategia de política exterior la encontramos en la Alternativa Bolivariana para las Américas (ALBA), una propuesta de unión política más que económica para construir un bloque latinoamericano de contrapoder que sea capaz de resistir y negociar en mejores términos y condiciones con el poder particularmente de los Estados Unidos.

La politización de la idea de integración regional, es el plus de la política petrolera del gobierno revolucionario liderado por Hugo Chávez. Es eso lo que constituye una absoluta novedad en las relaciones internacionales de Venezuela (MORA, 2009, p. 28).

Ahora bien, el hecho de que en Venezuela se estuviera construyendo un sistema político y económico socialista, generó un nuevo frente en la política exterior del país.

En este trabajo reconocemos que a partir del año 2004, Venezuela adquiere una nueva faceta en el ámbito internacional.

Actúa en su frente económico, como país exportador de petróleo y, como país revolucionario en su frente político, donde se compromete con la consolidación del proyecto socialista bolivariano anti imperialista.

Los dos frente a los que hacemos referencia consideran el petrolero como principal herramienta para la ejecución de proyectos y estrategias.

Entonces, la propuesta chavista de integración latinoamericana tiene como iniciativa más importante el ALBA. Bloque regional que sirve de techo para la propuesta de integración energética de la región, PETROAMERICA.

Tanto en la iniciativa del ALBA como en la de PETROAMERICA, Venezuela es contemplada como el país líder, el país financiador.

Para los formuladores de esa agenda internacional, el acervo energético que posee el país, posibilita el logro de una estrategia de desarrollo nacional que combine el uso soberano del recurso natural con la integración energética regional favorable al proyecto nacional revolucionario. Proyectando convertir a Venezuela en el mediano plazo en una potencia energética con influencia mundial (PPS, 2007, p.38). 
Ésta estrategia, en el Proyecto Nacional Simón Bolívar (2007-2013), aparece como corresponsable del desarrollo del nuevo modelo productivo socialista y, parte de la premisa del aumento de la probabilidad de que se "mantenga una tendencia irreversible al alza de los precios del petróleo crudo y del gas natural". No obstante, en el mismo párrafo, se prevé, "[...] ante la eventual persistencia de cierta volatilidad de los previos del petróleo crudo y del gas natural [...]", el desarrollo de una estrategia para diversificar, tanto como sea posible, las fuentes de recursos fiscales (PPS, 2007, p.38).

La premisa económica sobre la tendencia irreversible del alza de los precios del petróleo, consideramos, se aleja de la realidad, y constituye una gran debilidad estructural de la estrategia chavista.

Ahora bien, la defensa a la soberanía nacional, (que sustituye, en la constitución de 1999 a la defensa de la democracia representativa contemplada por el texto constitucional de 1961), según los formuladores de este PPS "nunca" constituye una conquista definitiva; la soberanía "siempre" hay que defenderla, o se corre el riesgo de perderla. Es por eso que se considera que, la soberanía de un país con la riqueza natural de hidrocarburos como Venezuela, "siempre" está amenazada (PPS, 2007, p. 39).

De manera que, en el entender de los formuladores del documento objeto de estudio, necesariamente, la política de Plena Soberanía Petrolera es una política internacional, de alianza con todos aquellos países que insisten en desarrollarse como naciones, y no aceptan marginarse en un mundo supuestamente globalizado (PPS, 2007, p. 38).

\subsection{LA ALTERNATIVA BOLIVARIANA PARA LAS AMÉRICAS (ALBA)}

En la III Cumbre de Jefes de Estado y de Gobierno de la Asociación de Estados del Caribe, celebrada en la isla de Margarita en diciembre de 2001, fue anunciada la creación de la Alternativa Bolivariana para las Américas, cuya base es la autodeterminación y la soberanía de los pueblos, y cuyos principios observan y buscan una alternativa al regionalismo abierto, para contrarrestar las medidas de políticas económicas propuestas e implementadas a finales del siglo pasado, durante las décadas de los ochenta y noventa por algunos organismos internacionales como el Banco Mundial y el Fondo Monetario Internacional (FLACSO, Dossier ALBA, 2007, p. 07).

El ALBA nace como alternativa a la propuesta integracionista liderada por Estados Unidos, el Área de Libre Comercio para las Américas. La cual, a finales de 2005 en la Cumbre de las Américas en Mar del Plata en Argentina, una parte importante de los Estados sudamericanos rehusó. Especialmente los gobiernos de Argentina, Brasil y Venezuela se negaron a retomar las negociaciones estancadas sobre el ALCA. Desde entonces, este proyecto central del gobierno estadounidense está congelado (FRIZT, 2007, p. 05).

Estas dos alternativas integradoras a la orden, llevó a que los gobiernos de la región se posicionaran al respecto, así existen: Estados que se mostraron favorables al modelo ALBA (Bolivia, Cuba, Ecuador, Nicaragua y Venezuela) y otros que no rechazan al ALBA de manera 
tajante, pero quieren liderar un proceso más parecido al de MERCOSUR (Argentina, Paraguay, Brasil y Uruguay) (MPPE, 2007, p. 8).

Sin embargo, en estos nueve países, hay un acuerdo en considerar que la propuesta estadounidense del Área de Libro Comercio para las Américas (ALCA) fracasó y fue "enterrada" en la Cumbre de Mar del Plata de 2005. En contraposición, existen gobiernos que se oponen de manera contundente al ALBA (Colombia, Chile, México y Perú), y se adhieren a las propuestas sostenidas por el gobierno estadounidense como lo son los Tratados de Libre Comercio (TLC) bilaterales (MPPE, 2007, p. 8).

\subsection{PETROAMERICA}

La integración energética de los países del subcontinente Sur Americano, es uno de los principales componentes del esfuerzo común de articulación de infraestructuras económicas nacionales (redes de transporte, telecomunicaciones, energía, etc.). Punto cardinal de la agenda consensual de la ahora denominada, Unión de Naciones Suramericanas (UNASUR) que substituyó la Comunidad Suramericana de Naciones (CASA) (SALOMÃO; MALHÃES, 2007).

Tal proceso tiene como punto de partida los proyectos binacionales implantados en el pasado, algunos durante gobiernos autoritarios, tales como la represa binacional de Itaipú e Yacyretá, los cuales dejaron lecciones de "cómo hacer" y "cómo no hacer"; esquemas de financiamiento apoyados en préstamos bancarios, falta de transparencia en la conducción de obras, sospechas de desvíos de recursos, resolución de la asimetrías en los acuerdos binacionales, etc. Otros fueron negociados por gobiernos democráticos, como por ejemplo, el gaseoducto Brasil-Bolivia y la producción de Gas por la PETROBRAS en el sudeste boliviano sin existir la adecuada articulación y ratificación de los respectivos congresos, generando problemas que se han hecho sentir (SALOMÃO; MALHÃES, 2007).

En el actual cuadro regional, nuevos proyectos de integración energética han sido propuestos. Teniendo PETROAMERICA, un papel importante.

En este trabajo asumimos que: PETROAMERICA es una idea en formación que evoluciona de acuerdo con las circunstancias que enfrentan los países de América Latina y el Caribe y con el desenvolvimiento de los mercados petroleros internacionales (MAYOBRE, 2005, p. 06).

Entonces, la propuesta del gobierno venezolano, fue inicialmente formulada como la creación de una empresa conjunta por parte de las empresas estatales de hidrocarburos de la región. En la medida que la misma se ha ido concretando se ha diversificado en mecanismos de alcance subregional, cada uno de ellos con características propias.

Así, Petrocaribe: abarca los países insulares del Caribe (con excepción de Trinidad y Tobago y Barbados), Guyana y Surinam. Petrosur: incluye a los países del MERCOSUR y, en Petroandina: participarían los Estados miembros de la Comunidad Andina de Naciones (CAN). 
En todos estos mecanismos, Venezuela participa con un papel financiador, en los términos y bajo las premisas establecidas en el Proyecto Nacional Simón Bolívar- Primer Plan Socialista de la Nación (2007-2013).

Las definiciones de esos mecanismos sub regionales, no son iguales entre sí, según el glosario de la estatal petrolera venezolana PDVSA, Petrosur: es un habilitador político y comercial, dirigido a establecer mecanismos de cooperación e integración sobre la base de la complementariedad y haciendo uso justo y democrático de los recursos energéticos para el mejoramiento socioeconómico de sus pueblos.

Petrocaribe, por su parte, es un acuerdo de cooperación energética, con el fin de resolver las asimetrías en el acceso a los recursos energéticos, por la vía de un nuevo esquema de intercambio favorable, equitativo y justo entre los países de la región caribeña.

Mientras tanto, Petroandina es una alianza estratégica de entes estatales petroleros y energéticos de los países de la CAN para impulsar la interconexión eléctrica y gasífera, la provisión mutua de recursos energéticos y la inversión conjunta en proyectos.

Como puede apreciarse entonces, PETROAMERICA es una iniciativa que subyace de la propuesta del ALBA, que representa un esquema de integración más amplio y que se divide por regiones. Mientras, que en Petrosur predominaría la complementariedad y la habilidad política y comercial, en Petrocaribe predomina la solidaridad y la cooperación y, en Petroandina la alianza estratégica estatal.

\section{CONSIDERACIONES FINALES}

En las páginas anteriores tuvimos la intención de hacer un panorama de la política externa venezolana, con especial atención al período de Hugo Chávez y a la idea de integración regional de su propuesta internacional.

Entonces, en nuestra investigación verificamos que la absoluta novedad introducida por Hugo Chávez en las relaciones internacionales venezolanas, es la politización de la política externa. Lo que en este trabajo denominamos de la nueva faceta que adquiere la identidad venezolana. La de país comprometido con los valores socialistas. Bajo esa concepción socialista de izquierda fueron planteadas iniciativas de integración regional que respondieran a ésos principios, donde el petróleo venezolano tiene un papel protagónico. No obstante, la premisa económica considerada al momento de la formulación de la agenda internacional, pone en duda la viabilidad del proyecto.

Notamos también que esa estrategia de política externa es un reflejo del modelo doméstico de desarrollo que estaba siendo instaurado en Venezuela, el Socialismo del Siglo XXI. Estudiamos como la acelerada construcción de dicho sistema económico, hizo con que la política externa adquiriera una nueva faceta. Además de la tradicional postura de país exportador de petróleo e importador de mercaderías. 


\section{BIBLIOGRAFIA}

CHÁVEZ, Hugo. Presentación de las Líneas Generales del Plan Nacional de Desarrollo Económico y Social de la Nación 2001-2007. In: Líneas Generales del Plan Nacional de Desarrollo Económico y Social de la Nación 2001-2007. República Bolivariana de Venezuela, sep. 2001, pp. 07-09.

LACSO, Dossier ALBA. San José: Publicaciones FLACSO, 2007.

FRIZT, Thomas. La Alternativa Bolivariana para las Américas: una nueva vía para la integración regional en Latinoamérica. Berlín: Centro de investigación y documentación Chile Latinoamérica, 2007.

GUERÓN, Eva. La Política Exterior: Continuidad y Cambio, Contradicción y Coherencia. In: NAIM, Moisés; PIÑANGO, Ramón (Org.). El Caso Venezuela: Una Ilusión de Armonía Caracas. Ediciones IESA, C.A, 1989, pp. 350-375.

MAYOBRE, Eduardo. La propuesta Petroamérica y la integración energética en América Latina y el Caribe. Caracas: Instituto Latinoamericano de estudios sociales, 2009.

MORA, Jesús. Las Bases de la Política Petrolera Rentista y Bolivariana del Gobierno de Chávez. Opiniones Contrapuestas, n. 05, 2009.

REPÚBLICA DE VENEZUELA. Constitución 1961.

REPÚBLICA BOLIVARIANA DE VENEZUELA, Líneas Generales del Plan Nacional de Desarrollo Económico y Social de la Nación 2001-2007. Caracas: Presidencia de la República, 2001.

REPÚBLICA BOLIVARIANA DE VENEZUELA, Proyecto Nacional Simón Bolívar Primer Plan Socialista 2007-2013. Caracas: Presidencia de la República, 2007.

REPÚBLICA BOLIVARIANA DE VENEZUELA. Constitución de 1999. Gaceta oficial 36.860, 1999.

ROMERO, Carlos. Crisis Política y Transiciones en Venezuela. Cadernos Prolam/USP. v.15, n. 27, 2015.

ROMERO, Carlos; ROMERO, María Teresa; CARDOZO, Elsa. Política Exterior en las Constituciones de 1961 y 1999: Una visión comparada de sus principios, procedimientos y temas. Revista venezolana de economía y ciencias sociales. v. 09, n. 01, 2003, pp. 163-183.

ROMERO, María Teresa. Política Exterior Venezolana: el proyecto democrático 1959-1999. Caracas: Libros el Nacional, 2002. 
REPÚBLICA BOLIVARIANA DE VENEZUELA. Constitución de 1999. Gaceta oficial 36.860, 1999.

TORO, José. Venezuela 55 años de Política Económica: 1936-1991 una utopía Keynesiana. Caracas: Editorial Panapo, 1992.

VIZENTINI, Pablo. A política externa da Venezuela frente à globalização (1989-2001). In: GUIMARAES, Samuel; CARDIM, Carlos (Org.). Venezuela: Visões brasileiras. Brasília: Instituto de Pesquisa de Relações Internacionais- IPRI, 2003, pp. 55-80 